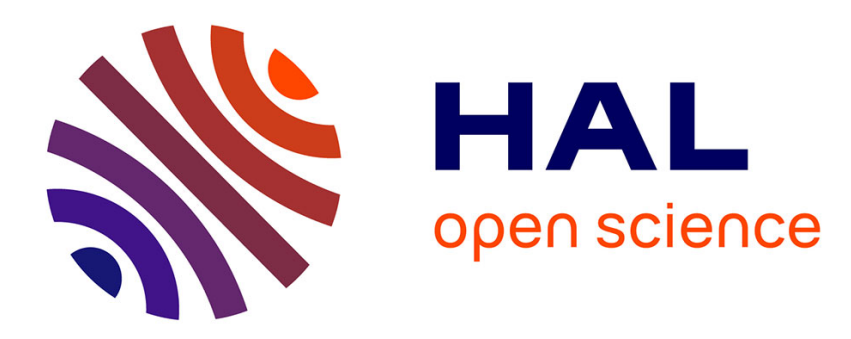

\title{
Architecture dirigée par les modèles pour une représentation multi-vues du contexte de coopération
}

\author{
Gilles Halin, Sylvain Kubicki
}

\section{To cite this version:}

Gilles Halin, Sylvain Kubicki. Architecture dirigée par les modèles pour une représentation multi-vues du contexte de coopération. 2006. hal-00086414

\section{HAL Id: hal-00086414 \\ https://hal.science/hal-00086414}

Preprint submitted on 19 Jul 2006

HAL is a multi-disciplinary open access archive for the deposit and dissemination of scientific research documents, whether they are published or not. The documents may come from teaching and research institutions in France or abroad, or from public or private research centers.
L'archive ouverte pluridisciplinaire HAL, est destinée au dépôt et à la diffusion de documents scientifiques de niveau recherche, publiés ou non, émanant des établissements d'enseignement et de recherche français ou étrangers, des laboratoires publics ou privés. 


\section{Architecture dirigée par les modèles pour une repré- sentation multi-vues du contexte de coopération}

\author{
Gilles Halin \\ MAP CRAI \\ 2, rue Bastien Lepage BP 40435 \\ 54001 Nancy Cedex, France \\ gilles.halin@crai.archi.fr
}

\author{
Sylvain Kubicki \\ MAP CRAI \\ 2, rue Bastien Lepage BP 40435 \\ 54001 Nancy Cedex, France \\ sylvain.kubicki@crai.archi.fr
}

\begin{abstract}
RESUME
Les outils visant à supporter les activités coopératives de conception-réalisation de produits doivent aujourd'hui intégrer le contexte afin de mieux répondre aux besoins des acteurs. La prise en compte du contexte permet de définir quelles sont les informations et les fonctionnalités dont l'utilisateur aura besoin, ou encore quelle interface est la plus adaptée à une situation donnée. Cet article décrit une architecture guidée par les modèles (MDA) qui permet l'intégration de plusieurs modes de visualisation en sélectionnant l'information dans le contexte de coopération. Nous proposons une application de cette méthode dans le domaine de l'architecture et de la construction.
\end{abstract}

MOTS CLES : Contexte, Mode de visualisation, Point de vue, Coopération, Modèle, MDA.

\begin{abstract}
Nowadays, new tools appear to assist the cooperative activities of design and realisation. These tools have to integrate the context to better answer to the users' needs. Taking into account the context allows to define relevant information and functionalities for the user, or to suggest adapted interfaces for specific needs. This article describes a model driven architecture (MDA) allowing the integration of different visualisation modes by selecting information in the cooperative context.
\end{abstract}

CATEGORIES AND SUBJECT DESCRIPTORS: H.5.2 [User Interfaces]: User-centered design.

GENERAL TERMS: Design, Theory

KEYWORDS: Context, Visualisation mode, Point of view, Cooperation, Model, MDA.

Réserver cet espace pour la notice de copyright

\section{INTRODUCTION}

Les activités coopératives de conception-construction réunissent de nombreux acteurs coopérant autour d'un projet avec un objectif commun. La complexité de ces situations de coopération conduit depuis quelques années au développement d'outils d'assistance. La prise en compte du contexte est un facteur essentiel pour le développement de ces outils et leur utilisation. La modélisation du contexte coopératif et la définition de points de vue d'acteurs est étroitement liée à la proposition de modes de visualisation adaptés au contexte.

Nous proposons une architecture guidée par les modèles (MDA [1]) permettant de construire des modes de visualisation en sélectionnant les informations dans le contexte coopératif.

Nous décrivons enfin une application possible de ces propositions dans le domaine de l'architecture et de la construction. Ce secteur de production est en effet marqué par une coopération éphèmère et évolutive entre de multiples acteurs issus de milieux variés. Les modes de visualisation disponibles dans les outils d'assistance au chantier doivent donc s'adapter au contexte des acteurs et à leurs besoins...

\section{CONTEXTE ET ACTIVITE COOPERATIVE}

La définition générale du contexte est l'ensemble des circonstances dans lesquelles s'insère un fait [Petit Robert 1992].

Dans les différents domaines d'application, on retrouve une définition relativement stable. Le contexte est un ensemble d'informations, relatives à une interaction entre agents. En IHM, il intervient dans l'interaction entre l'homme et la machine. Dans le développement d'applications «context-aware », on élargit les agents à l'environnement d'utilisation d'un outil [2].

Au-delà de ces définitions, on constate que les perspectives portées par la prise en compte du contexte conditionnent fortement son degré d'implication dans l'interaction. Brézillon [3] distingue 3 perspectives :

- La perspective « informationnelle » où le contexte fournit un espace d'information adapté en fonction du contexte de l'utilisateur et complété par des outils et processus,

- La perspective «fonctionnelle » dans laquelle le contexte conditionne les fonctions utiles des outils 
relativement à des interactions identifiées et à l'espace physique des pratiques. Le contexte intègre et permet la diffusion de l'information qui assiste le travail humain,

- La perspective « infrastructurelle » où le contexte est pris en compte dans les outils qui manipulent de l'information sur l'environnement fournie par d'autres composants du système.

Les interactions entre agents définissent le contexte le plus général, dans lequel sont référencés ou décrits les autres contextes [3]. Dans le cadre de la conduite d'un projet, le contexte de coopération contient toutes les entités en interaction dans le projet : acteurs, activités, documents et objets (Figure 1).

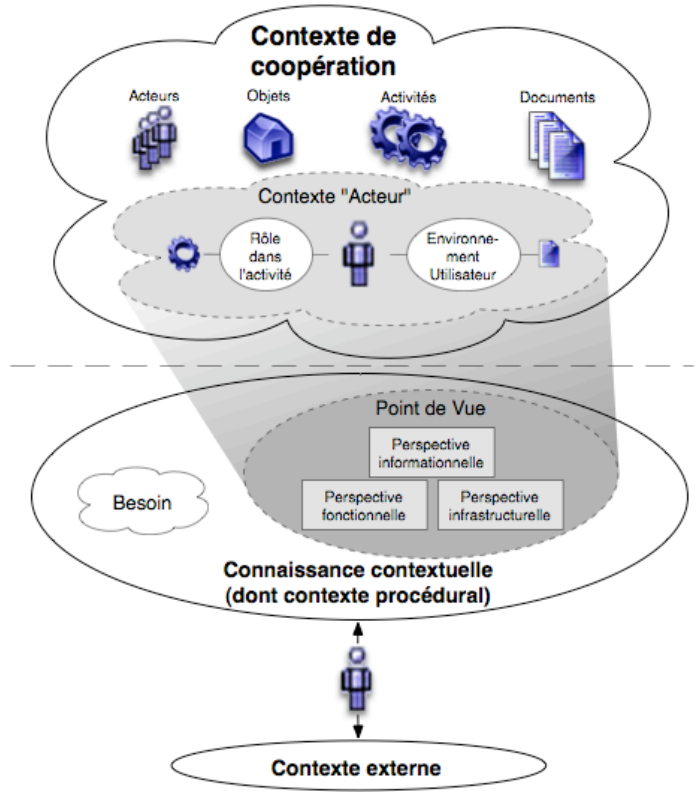

Figure 1: Contexte de coopération et contexte « acteur ».

Le contexte d'un acteur coopérant dans le projet est une restriction du contexte de coopération. Son rôle dans l'activité et les informations sur son environnement permettent de définir son point de vue sur le contexte. Le rôle de l'acteur dans l'activité collective permet de définir en amont l'étendue du contexte qu'il peut visualiser (droits, visibilité sur l'information). L'environnement de l'utilisateur permet aussi de restreindre son contexte. Son implication dans une activité participe de son potentiel d'action. Les moyens disponibles sont aussi essentiels (matériel utilisé, lieu d'utilisation etc.).

La réflexion sur les interfaces entre utilisateur et système nous conduit à centrer la question du contexte sur l'acteur dans le cadre d'une activité coopérative.

Selon Gachet et Brézillon [4], un acteur d'un système mobilise des connaissances contextuelles sur l'activité en cours, dont une partie est liée aux procédures mises en oeuvre pour réaliser un tâche spécifique (contexte procédural). Nous considérons que cette connaissance contextuelle est liée d'une part au point de vue de l'individu sur l'activité coopérative, et d'autre part à son besoin (Figure 1). A cela s'ajoute le contexte externe, propre à chaque acteur (ex connaissances, expérience...). Les perspectives dégagées par la représentation du contexte de l'acteur pour le développement d'outils « sensibles au contexte » sont de 3 ordres :

- Informationnel : informations adaptées au besoin de l'acteur et liées à son point de vue,

- Fonctionnel : fonctions et outils proposées à l'utilisateur en fonction de son potentiel d'action, lui aussi lié au point de vue,

- Infrastructurel : interfaces et matériels fournis à l'utilisateur pour accomplir son action en fonction de son besoin [5].

Ces perspectives se traduisent par des fonctions ou des types d'informations dans des applications « sensibles au contexte ». Enfin, Rosa et al. [6] dans leur analyse sur l'utilisation du contexte dans les « groupwares » définissent celui-ci comme étant l'ensemble des informations sur les acteurs (individuels et groupes), les tâches planifiées, les relations entre acteurs et tâches, les tâches achevées et l'environnement.

\section{REPRESENTATION DU CONTEXTE COOPERATIF}

Toute coopération s'inscrit dans un domaine particulier dans lequel les acteurs, les documents, les activités, les règles et l'objet de la coopération sont différents. Seule les concepts agissant et interagissant dans cette coopération sont communs. Ainsi toute coopération, et donc son contexte, peut être décrite par un ensemble d'acteurs, d'activités, et d'artefacts utilisés pour décrire l'objectif de la coopération (documents et représentations de l'objectif). La représentation du contexte de coopération que nous proposons repose sur l'architecture MOF par la proposition d'un méta-modèle décrivant ces concepts et leur relation. Une modèle permettant la description d'un contexte de coopération dans un domaine précis (conception d'un bâtiment, d'une voiture ou d'un logiciel) est alors une instanciation de ce méta-modèle [5].

Quatre classes représentent les concepts généraux de notre meta-modèle : acteur, activité, document et objet. Ces concepts sont également liés entre eux par des relations. La relation Acteur-Activité est de premier ordre dans ce modèle, car elle participe à la représentation du rôle joué par un acteur dans une activité (qui peut faire quoi ?) et du rôle qu'a joué un acteur dans une activité (qui a fait quoi ?). L'instanciation du méta-modèle dans le domaine de la conception d'un bâtiment permettra d'énoncer l'ensemble des phases d'un projet (Esquisse, AvantProjet Sommaire (APS), Avant-Projet Définitif (APD), le chantier etc.), l'ensemble des acteurs potentiels (le maître d'ouvrage, le maître d'œuvre, les entreprises...), les documents (permis de construire, descriptif d'exécution...) et enfin l'objet (les ouvrages à réaliser, les espaces) [5]. Le modèle obtenu décrit alors le contexte de coopération d'une activité de conception/construction. Le contexte de coopération (CC) du projet d'un bâtiment particulier constitue une instanciation de ce dernier modèle. 


\section{POINT DE VUE ET VISUALISATION}

La visualisation d'un point de vue d'un acteur sur ce contexte de coopération va alors être réalisé par un ou plusieurs outils participant à la perspective infrastructurelle du contexte de chaque acteur. Chaque outil met en œuvre un mode de visualisation particulier permettant à l'acteur de comprendre puis d'interagir sur le contexte présenté. La perspective informationnelle d'un mode de visualisation est l'ensemble des données qui doivent être visualisées. L'ensemble des opérations réalisables constitue alors la perspective fonctionnelle. Ces deux perspectives peuvent être décrites dans un modèle associé au mode de visualisation concerné. La construction du point de vue peut être considéré comme une transformation de modèles où le modèle d'origine est le modèle décrivant le contexte coopératif (MCC) et le modèle de destination celui qui décrit le contexte de l'acteur dans un mode de visualisation particulier (MMV).

Ces transformations de modèles peuvent s'inscrire dans l'architecture MDA du MOF et être représentées sous la forme d'une pyramide où le contexte acteur des différents modes de visualisation sont situés aux extrémités de la base et le contexte de coopération au centre (Figure 2). La sélection des données du contexte de coopération vers un contexte acteur est alors modélisée au niveau supérieur de la pyramide par la spécification d'une transformation du MCC vers le MMV concerné.

Dans cette architecture, chaque modèle possède son méta-modèle (qui peut être commun à plusieurs modèles). Ainsi le méta-modèle d'une transformation décrira la forme des règles à instancier pour décrire une tranformation du MCC vers un MMV.

Nous pouvons illustrer cette architecture en prenant comme exemple la visualisation du contexte acteur de l'outil Bat'Map [7]. Le mode de visualisation de cet outil propose un graphe interactif où les nœuds sont des acteurs, des documents, des activités, et des ouvrages (Jean Cartier <Nœud Acteur>, PC n546 <Nœud Document>) et où les liens matérialisent les rélations entre les entités du projet (a produit [Jean Cartier, $\mathrm{PC} \mathrm{N}^{\circ} 546$ ] < LienProducteur [Nœud Acteur, Nœud Document]>). A chacun des éléments du graphe sont associés une liste d'actions que peut réaliser l'acteur en fonction de son rôle dans le projet. Le modèle de ce mode de visualisation définit l'ensemble des types de nœuds, des types de liens dont a besoin Bat'Map pour représenter le contexte d'un projet et aussi l'ensemble des opérations possibles sur chacun des éléments du graphe (Nœud Acteur $<$ Nœud $>$, Nœud Document $<$ Nœud $>$, LienProducteur $<$ Lien $>$ ). Le metamodèle quant à lui décrit en MOF la structure d'un graphe interactif (Nœud, Lien, Opérations).

La construction du contexte acteur s'effectue en sélectionnant les entités du contexte de coopération (CC) nécessaires pour la visualisation tout en respectant le modèle de transformation définit au niveau des modèles. Ce modèle est un ensemble de règles décrivant les transformations des entités du MCC vers les entités du MMV (Acteur -> NœudActeur, Document -> NœudDocument).

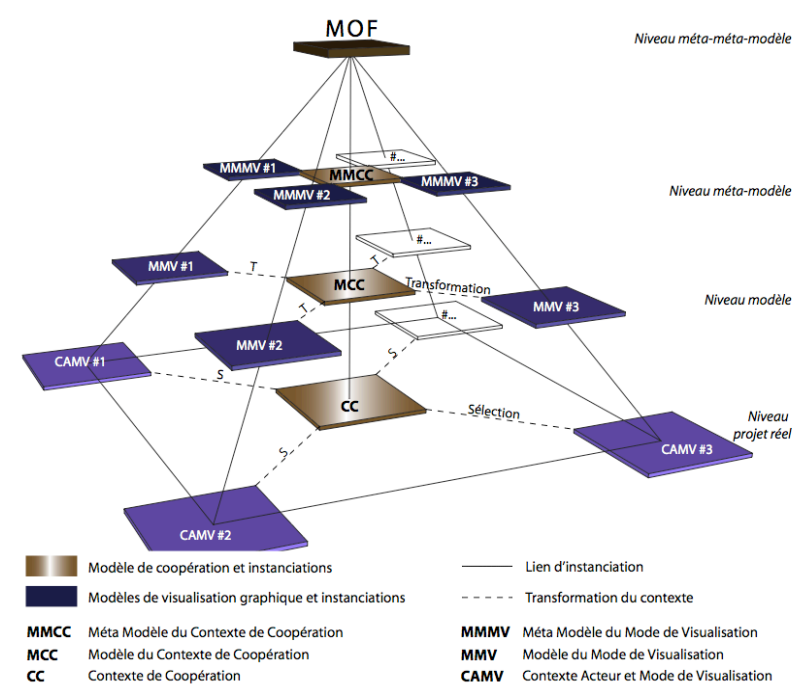

Figure 2 : Transformation et visualisation du contexte.

\section{APPLICATION AU SUIVI DE CHANTIER}

Notre champ d'application est celui des activités coopératives liées au déroulement du chantier de construction. L'activité de construction peut être comparée à d'autres activités de réalisation industrielles, en effet les tâches de construction sont identifiées et ordonnancées. Cependant, la nature même du projet architectural distingue sa réalisation des autres secteurs industriels. Le produit «bâtiment » est unique et ne sera réalisé qu'en un seul exemplaire. De plus, les équipes de construction sont indépendantes de la maîtrise d'oeuvre et il est très difficile d'anticiper les problèmes de mise en oeuvre. Notre étude se focalise sur l'activité coopérative durant le chantier. La coordination prédictive (ordonnancement en amont de la réalisation) et réactive (ajustement, suivi du chantier) sont des activités coopératives majeures dans la conduite du projet.

Les acteurs responsables du pilotage et de la coordination du chantier disposent de divers outils et méthodes :

- Les documents textuels ou tabulaires sont les plus utilisés actuellement pour diffuser l'information,

- Les plans 2D sont utilisés pour représenter le projet,

- Les méthodes de plannification intègrent le temps et permettent de représenter les tâches.

- Les outils de CAO permettent de réaliser des maquettes numériques du projet (3D),

- Des outils «4D» associent de l'information sur l'exécution des tâches aux objets de la maquette ...

À ces modes de visualisation s'ajoute le graphe, idéal pour représenter le contexte organisationnel et les relations entre les entités du contexte de coopération.

Ce secteur d'activité est donc riche et complexe. Les pratiques des acteurs et leurs besoins sont très disparates et les informations concernant le contexte sont dispersées entre les acteurs et les applications qu'ils utilisent.

Ces outils présentent des limites qui peuvent être à l'origine de problèmes de coordination (surcharge informationnelle, pas de personnalisation de l'information 
etc.). Chaque acteur devrait obtenir l'information utile en fonction de son contexte et de sa place dans le contexte coopératif. Nous distinguerons par exemple les rôles du maître d'ouvrage (client), de l'architecte (coordinateur) ou de l'entrepreneur (réalisateur). Les données environnementales sur l'utilisateur dans un projet de construction sont aussi utiles dans la diffusion de l'information, par exemple les relations contractuelles entre les acteurs (sous-traitance) ou les outils utilisés (ordinateur, PDA) et les lieux d'utilisation (bureau, cabane de chantier)...

Dans le domaine du bâtiment, les différentes perspectives d'utilisation du contexte de l'acteur, énoncées dans la partie 1, permettent d'imaginer des outils adaptatifs :

- Perspective informationnelle: déterminer l'information pertinente en fonction de l'utilisateur d'un outil. Par exemple, l'architecte a un point de vue global sur l'information du projet alors que l'entreprise ne doit voir que ce qui la concerne,

- Perspective fonctionnelle : anticiper les besoins de l'utilisateur d'un outil. Dans le cas du compte rendu de réunion, le coordonnateur de chantier aura besoin de fonctionnalités de rédaction ou de recherche alors que les autres acteurs récupéreront et liront simplement le document,

- Perspective infrastructurelle : proposer des interfaces de visualisation adaptées (Figure 3). La complexité du contexte de coopération en architecture et la variété des données manipulées nous conduisent à envisager des interfaces adaptées à l'acteur (son rôle) et au besoin qu'il formule (données environnementales). Par exemple, le coordinateur de chantier travaille en général avec le planning d'exécution des tâches alors que l'architecte préférera visualiser le projet dans l'espace (3D)...

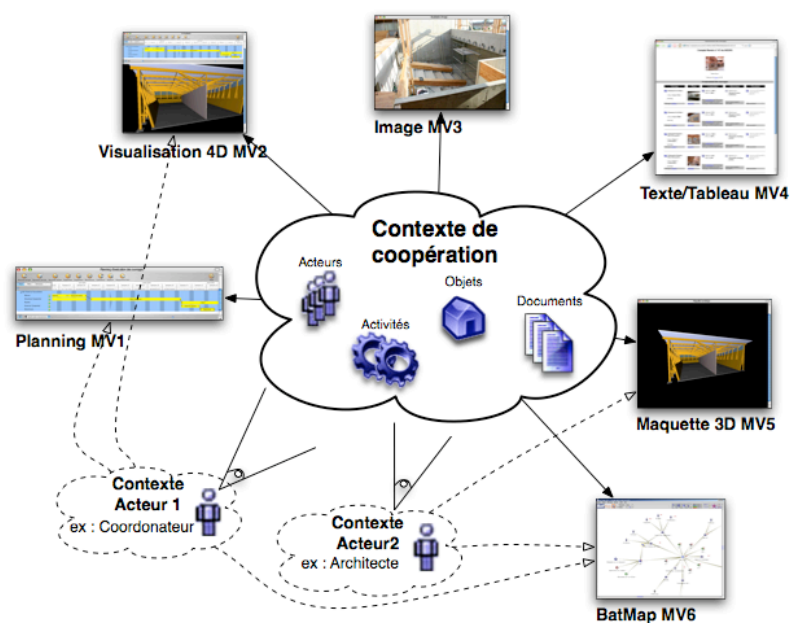

Figure 3 : Modes de visualisation adaptés au contexte.

\section{CONCLUSION}

La complexité des interactions entre agents dans le cadre d'une activité coopérative peut être surmontée par la prise en compte du contexte dans les applications d'assistance à la coopération et la proposition de modes de visualisation adaptés à chacun des utilisateurs.

Chaque mode de visualisation étant descriptible par un modèle, nous proposons d'intégrer ces modèles dans une architecture MDA dans laquelle un modèle de description du contexte de coopération occuperait la place centrale. C'est à partir de cette architecture que nous souhaitons développer un ensemble de services web offrant à chacun des outils de visualisation le contexte acteur adéquat.

L'interopérabilité entre applications est donc une composante essentielle de la réflexion. Au-delà de la transformation de modèles entre les applications, la composition même de ces modèles doit s'appuyer sur une sémantique commune. Dans le secteur du bâtiment, la spécification IFC développée par l'IAI [8] propose un format de description partagé des objets du projet.

Enfin, le domaine de l'architecture et de la construction englobe une variété d'acteurs impliqués dans l'activité coopérative. La diversité de ces points de vue nécessite des modes de visualisation adaptés aux besoins et au rôle de chacun des acteurs afin d'augmenter leur perception du contexte de coopération et ainsi leur implication dans le projet.

\section{BIBLIOGRAPHIE}

1. Blanc, X. MDA, Ingénierie logicielle guidée par les modèles. Eyrolles éditions, Paris, 2005.

2. Dey, A.K. and Abowd, G.D. Towards a Better Understanding of Context and Context-Awareness. In 1st international symposium on Handheld and Ubiquitous Computing. Springer-Verlag, Karlsruhe, Germany, 1999.

3. Brézillon, P. Hors du contexte, point de salut. In Séminaire "Objets Communicants". Autrans, France, 2002.

4. Gachet, A. and Brézillon, P. A context-based representation of organizational structures. In FLAIRS Conference. Clearwater Beach, Florida, 2005.

5. Halin, G., Hanser D., and Bignon, J.C. User Adaptative Visualization of Cooperative Architectural Design. In International Journal of Architectural Computing. Vol. 01, No.02, 2003, p. 89-107.

6. Rosa, M.G.P., Borges, M.R.S., and Santoro, F.M. A conceptual framework for analyzing the use of context in groupware. In CRIWG Conference. Springer Editions, Autrans, France, 2003.

7. Halin, G. and Hanser D. Vers une visualisation contextuelle de la conception coopérative. In IHM Conference. Caen, France, 2003.

8. http://www.iai-international.org 